\title{
Hypermethylation of the alternative AWT1 promoter in hematological malignancies is a highly specific marker for acute myeloid leukemias despite high expression levels
}

\author{
Amy Guillaumet-Adkins ${ }^{1}$, Julia Richter ${ }^{2}$, Maria D Odero ${ }^{3}$, Juan Sandoval ${ }^{4}$, Xabi Agirre ${ }^{5}$, Albert Catala ${ }^{6}$,
} Manel Esteller ${ }^{4,7,8}$, Felipe Prósper ${ }^{5}$, María José Calasanz ${ }^{3}$, Ismael Buño ${ }^{9}$, Mi Kwon ${ }^{9}$, Franck Court ${ }^{1}$,

Reiner Siebert ${ }^{2}$ and David Monk ${ }^{1 *}$

\begin{abstract}
Background: Wilms tumor 1 (WT1) is over-expressed in numerous cancers with respect to normal cells, and has either a tumor suppressor or oncogenic role depending on cellular context. This gene is associated with numerous alternatively spliced transcripts, which initiate from two different unique first exons within the WT1 and the alternative (A)WT1 promoter intervals. Within the hematological system, WT1 expression is restricted to CD34+/ CD38- cells and is undetectable after differentiation. Detectable expression of this gene is an excellent marker for minimal residual disease in acute myeloid leukemia (AML), but the underlying epigenetic alterations are unknown.

Methods: To determine the changes in the underlying epigenetic landscape responsible for this expression, we characterized expression, DNA methylation and histone modification profiles in 28 hematological cancer cell lines and confirmed the methylation signature in 356 cytogenetically well-characterized primary hematological malignancies.

Results: Despite high expression of WT1 and AWT1 transcripts in AML-derived cell lines, we observe robust hypermethylation of the AWT1 promoter and an epigenetic switch from a permissive to repressive chromatin structure between normal cells and AML cell lines. Subsequent methylation analysis in our primary leukemia and lymphoma cohort revealed that the epigenetic signature identified in cell lines is specific to myeloid-lineage malignancies, irrespective of underlying mutational status or translocation. In addition to being a highly specific marker for AML diagnosis (positive predictive value 100\%; sensitivity $86.1 \%$; negative predictive value $89.4 \%$ ), we show that AWT1 hypermethylation also discriminates patients that relapse from those achieving complete remission after hematopoietic stem cell transplantation, with similar efficiency to WT1 expression profiling.
\end{abstract}

Conclusions: We describe a methylation signature of the AWT1 promoter CpG island that is a promising marker for classifying myeloid-derived leukemias. In addition AWT1 hypermethylation is ideally suited to monitor the recurrence of disease during remission in patients undergoing allogeneic stem cell transfer.

Keywords: WT1, Methylation, Epigenetics, Leukemia, Imprinting

\footnotetext{
* Correspondence: dmonk@idibell.cat

${ }^{1}$ Imprinting and Cancer group, Cancer Epigenetic and Biology Program,

Institut d'Investigació Biomedica de Bellvitge, Hospital Duran i Reynals,

Av. Gran Via de L'Hospotalet 199-203, 08907 L'Hospitalet de Llobregat,

Barcelona, Spain

Full list of author information is available at the end of the article
} 


\section{Background}

Overexpression of the Wilms' tumor 1 gene (WT1) is implicated in the prognosis of leukemia with high expression predicting disease progression in acute myeloid leukemia (AML), as well as being intensively studied as a potential molecular marker for minimal residual disease (MRD) and treatment response. In the normal scenario, WT1 expression is restricted to CD34+ cells in the bone marrow and absent in peripheral blood cells; therefore expression of this zinc-finger transcription factor is limited to early progenitors of the blood system, suggesting that this gene plays a critical role in controlling proliferation and/or differentiation in hematopoietic stem cells [1].

Many different isoforms exits for WT1, and it is estimated that it can have over 36 different isoforms generated by alternative transcription initiation, mRNA splicing and alternative translation initiation [2]. The most studied and common splicing events affects exon 5 leading to the presence or absence of 17 amino acids $(+17 /-17 \mathrm{aa})$ and alternative-splicing within exon 9 with the possible insertion of three amino acids (lysine, threonine and serine: $+\mathrm{KTS} /-\mathrm{KTS}$ ), resulting in a $52-54 \mathrm{kDa}$ protein. Recently an alternative promoter incorporating a unique first exon has been described. The alternative WT1 transcript $(A W T 1)$ encodes a $32 \mathrm{kDa}$ protein deficient for the first 147 amino acids of the N-terminal of the WT1 protein, which lacks the repressor domain and the RNA recognition motif of the full-length protein [3]. Transcripts from this alternative promoter also undergo splicing events leading to the $+/-17$ aa and $+/-$ KTS isoforms. In addition to these protein-coding transcripts, an non-coding antisense transcript (WT1-AS) also originates within the $\sim 5 \mathrm{~kb}$ promoter interval (Figure 1A). Both the antisense and $A W T 1$ transcripts have been reported to be paternally expressed in kidney and blood samples $[4,5]$. These transcripts originate from a region of partial methylation, the WT1 antisense regulatory region (WT1-ARR) that has been proposed to act as a cisacting transcriptional silencer on the maternal allele [6].

Expression profiling of WT1 is becoming almost universal in characterizing AML. Recently, raised WT1 levels at diagnosis and post-induction are associated with poorer outcomes, whereas patients with very low WT1 level post-intensification had excellent outcomes [7]. Despite aberrant expression of WT1 being a useful marker for diagnosis and monitoring MRD in AML the underlying epigenetic alterations associated with this expression are unknown [8]. Using a panel of 28 hematological cancer cell lines and more than 350 primary samples we identify AML-specific $A W T 1$ promoter hypermethylation that is present irrespective of underlying translocation/oncogenic fusion protein or mutations, which is accompanied by a concomitant epigenetic switch at the level of post-translational histone tail modifications. Finally we show that this robust $A W T 1$ epigenetic signature is an excellent marker for discriminating AML from non-diseased peripheral blood and that this hypermethylation signature can accurately track disease progression, differentiating patients who relapse from those achieving complete remission after allogeneic hematopoietic stem cell transplantation $(\mathrm{SCT})$.

\section{Results}

\section{Abundant biallelic expression of the WT1/AWT1}

transcripts result in nuclear retained proteins in myeloid derived leukemic cell lines

qRT-PCR was performed on a wide range of leukemia and lymphoma cell lines to evaluate the transcription levels of the WT1, AWT1 and the non-coding antisense transcript WT1-AS. In all myeloid origin cell lines evaluated the WT1 and $A W T 1$ transcripts were readily detectable, with high correlation (Pearson correlation, $\mathrm{r}=0.90$ ), indicating that they are likely to be co-regulated. The WT1-AS transcript was also expressed in most of the myeloid cell lines (Figure 1B). In contrast, the majority of B-, T- cell leukemic cell lines and lymphomas did not express these transcripts, with the exception of MOLT4 and NALM-20, the later being a cell line with a $t(9 ; 22)$ translocation and biphenotypic B-cell and myeloid cell characteristics [9]. The transcripts within the WT1 locus have been described to be expressed from the paternal allele in an imprinted manner [6,7]. The over-expression of these transcripts in the cell lines allowed us to determine allelic expression. Using primer pairs designed to distinguish individual isoforms (i.e. the forward primer was in the unique first exon of each transcript) and incorporate SNPs identified in the UCSC sequence browser (GRC27/hg19dbSNP 135) into the final RT-PCR product, we observe that WT1, AWT1 and WT1-AS are biallelically expressed in all heterozygous cell lines (Figure 1C). Finally, western blotting using an antibody directed against the C-terminal of the WT1 protein revealed that the abundant RNA levels for WT1 and AWT1 isoforms are translated into nuclear retained proteins in the myeloid derived cell lines KG1A, NB4 and K562, consistent with their transcription factor function (Figure 1D).

\section{AWT1 promoter hypermethylation despite over-expression in AML}

Having confirmed the high expression of WT1 and AWT1 in myeloid derived cancer cell lines we next wished to determine if this expression profile was associated with lineage specific DNA methylation changes. We determined the methylation profile of the WT1 $5^{\prime}$ interval using publically available WGBS datasets for CD34+ cells and peripheral leukocytes (Figure 2A). In addition we performed a specific bisulphite PCR analysis across the 


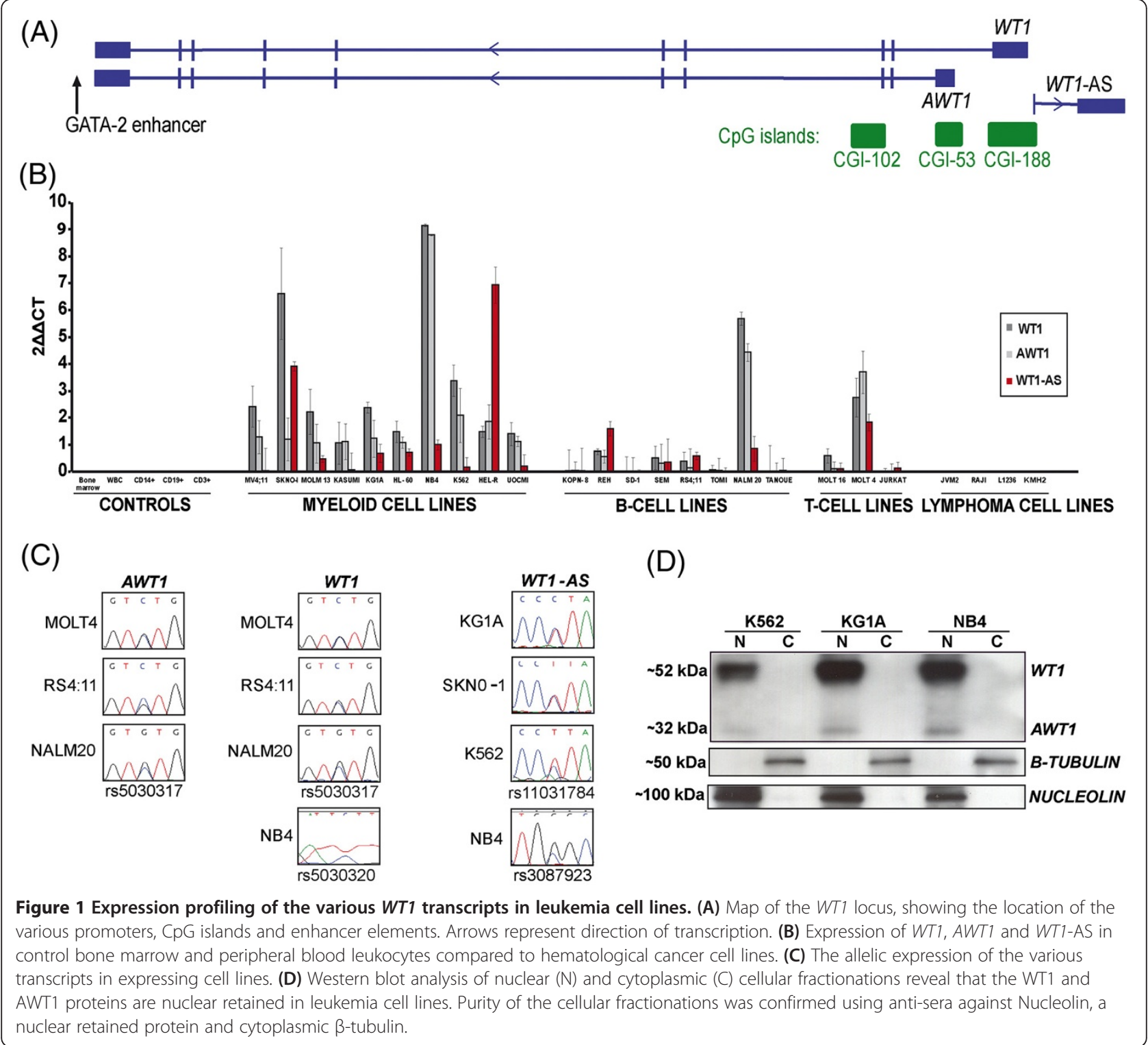

promoter interval in cell lines, peripheral blood leukocytes and immunosorted CD15+, CD19+ and CD3+ cells.

The promoter region for WT1 is located within a CpG island of $2.4 \mathrm{~kb}$ that is unmethylated in all normal cell types as revealed by the WGBS (chr11: 32454875 $32457311=\mathrm{CD} 34+3 \%$ methylated; leukocytes $5 \%$ methylated). Approximately $4 \mathrm{~kb}$ downstream in intron 1 , a second promoter region is embedded within an unmethylated CpG island of 560 bp (chr11: 32452145$32452708=$ CD34+ 24\% methylated; leukocytes 15\% methylated), giving rise to the AWT1 and WT1-AS isoforms (Figure 2A). Adjacent to these promoters is a region of proposed allele specific DNA methylation, the WT1ARR, which has been implicated in regulating expression of these transcripts (chr11: 32454083-32454765 = CD34+ $33 \%$ methylated; leukocytes $18 \%$ methylated). Our analysis utilizing standard bisulphite PCR and subcloning of normal leukocyte samples heterozygous for the SNP rs11031781 revealed that this region is mosaically methylated on both alleles (Figure 2B), consistent with a lack of imprinted methylation despite normal leukocytes having methylation on the paternal allele at the $H 19$ loci (Additional file 1: Figure S1A).

Methylation analysis of the entire panel of leukemia and lymphoma cell lines, determined by pyrosequencing, revealed that in myeloid derived cancer cell lines the WT1 CpG island promoter is unmethylated, except for the HL-60 cell line that was completely hypermethylated, whereas the AWT1 CpG island promoter was methylated in all cell lines evaluated. The WT1-ARR region showed varying amounts of methylation (Figure 2C). In B- and T-cell derived leukemic cell lines the WT1 


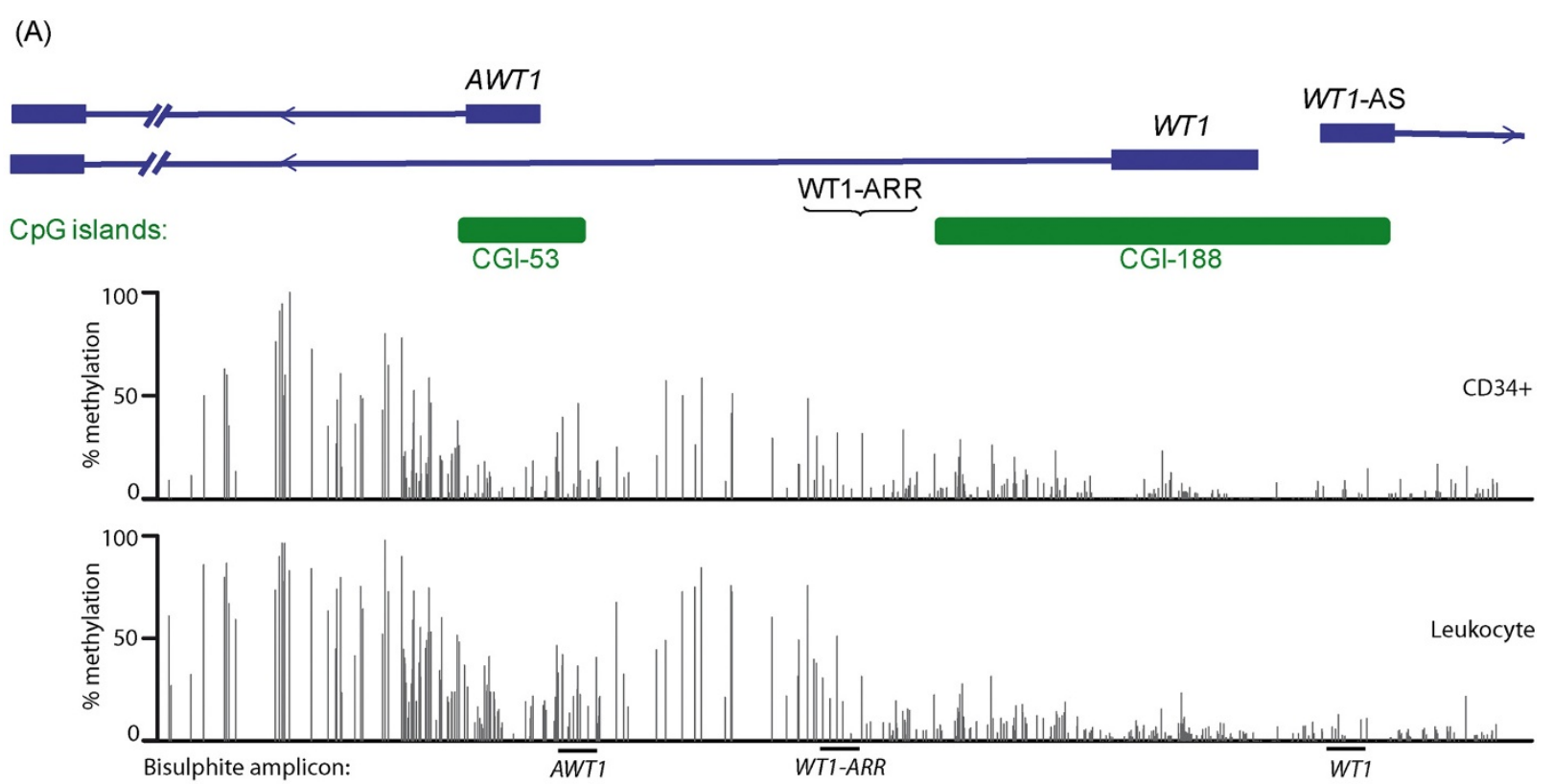

(B)

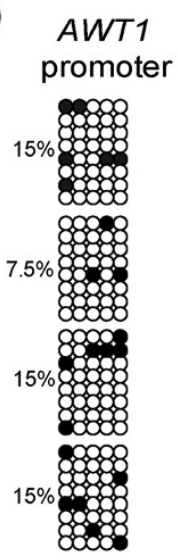

(D)
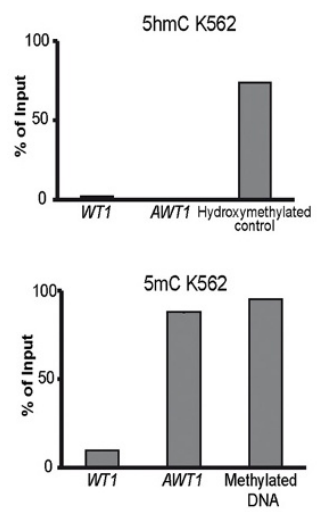
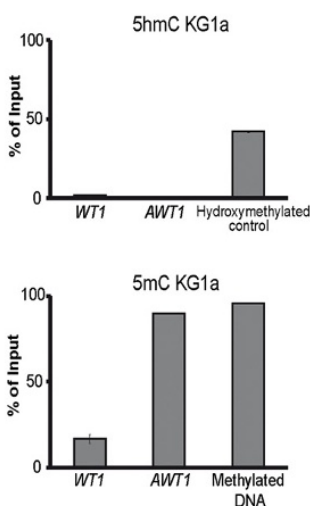

WT1

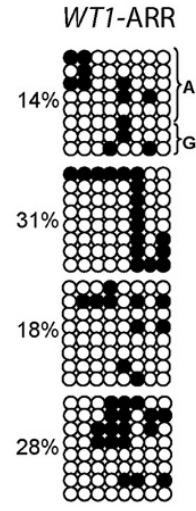

promoter

0\%

0600030

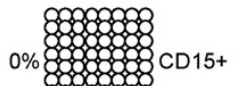

06060

11\%

(3)

89890969

$12 \%$ os

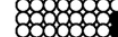

(C)

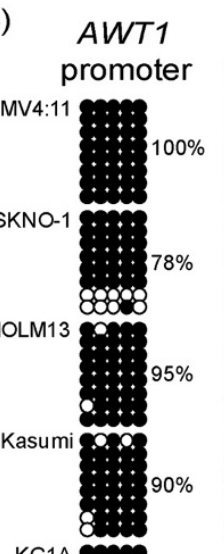

WT1

WT1-ARR promoter
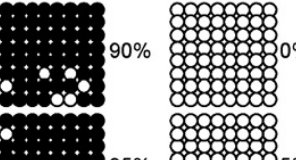

::: $: 05 \%$

: : : : : : 800808080

ㄱ: $8: 2800000$

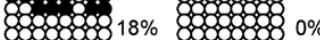

038000080
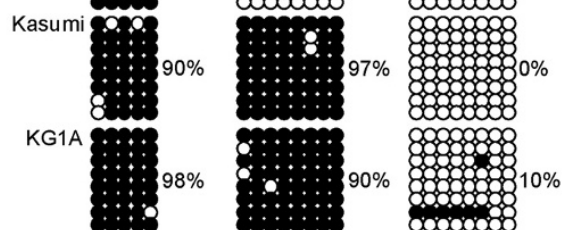

u०CM1

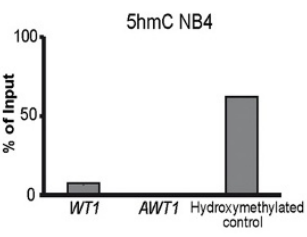

UOCM1
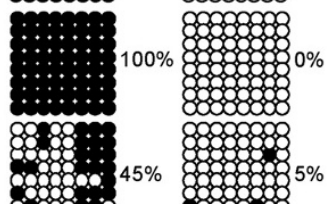

$100 \%$

80008.

00000.

\&mom

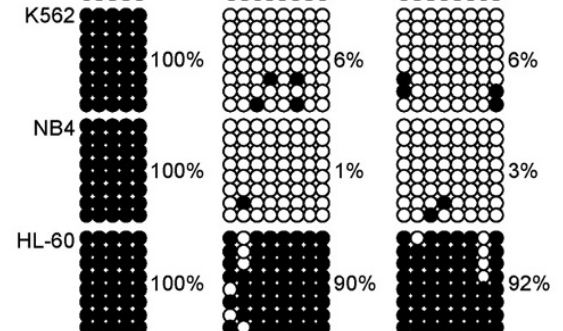

Figure $\mathbf{2}$ (See legend on next page.) 
(See figure on previous page.)

Figure 2 Methylation analysis in myeloid-derived cancer cell lines. (A) A detailed map of the WT1 promoter interval with the methylation profile for CD34+ and leukocyte cells determined by WGBS. Vertical dark grey lines in the WGBS tracks represent the mean methylation value for individual CPG dinucleotides calculated from multiple data sets, with the light grey lines representing the mean + standard deviation. The positions of the bisulphite PCR products are indicated. (B) The methylation status of the WT1 and AWT1 promoters and the WT1-ARR region in control leukocytes and immuno-selected cells. Each circle represents a single CpG dinucleotide on a DNA strand, a methylated cytosine (•) or an unmethylated cytosine (o). Allelic methylation was determined in all heterozygous samples. (C) The methylation profile in AML cell lines. (D) Quantitative PCR on meDIP preciptations using specific antibodies against $5 \mathrm{hmC}$ and $5 \mathrm{mC}$ in three cell lines that show hypermethylation of the AWT1 CpG island using bisulphite techniques.

promoter was frequently hypermethylated as was the $A W T 1$ promoter. In lymphoma cell lines, the entire locus was fully methylated (Additional file 1: Figure S1B).

\section{The AWT1 methylation detected by bisulphite analysis is $5 \mathrm{mC}$ and not $5 \mathrm{hmC}$}

Recently 5-hydroxymethylation $(5 \mathrm{hmC})$, an oxidized derivative of 5-methylcytosine $(5 \mathrm{mC})$ has been suggested to regulate gene expression and is moderately enriched within CpG rich promoters and actively transcribed gene bodies [10]. Unfortunately the deamination of unmethylated cytosines that occurs during conventional bisulfite reactions does not distinguish between $5 \mathrm{mC}$ and $5 \mathrm{hmC}$, therefore the bisulphite resistant cytosines we observed in the AML cell lines may reflect enrichment of $5 \mathrm{hmC}$ and not $5 \mathrm{mC}$. In order to differentiate these methylation states, we performed DNA immunoprecipitation utilizing antibodies that can discriminate $5 \mathrm{hmC}$ from $5 \mathrm{mC}$ in the myeloid cell lines, K562, KG1A and NB4. Using this technique we show that the methylation present at the $A W T 1$ promoter is $5 \mathrm{mC}$ (Figure 2D). We confirm these observations using a second method utilizing a highly specific $5 \mathrm{hmC}$ glucosyltransferase that tags a glucose moiety to $5 \mathrm{hmC}$ but not to $5 \mathrm{mC}$, rendering $\mathrm{h} 5 \mathrm{mC}$ refractory to digestion with the Glucosyl-5hmC Sensitive Restriction Endonucleases (GSRE) enzyme (MspI-CCGG) (data not shown).

\section{AWT1 hypermethylation is associated with a switch from permissive to repressed chromatin state}

Using publically available ChIP-seq datasets from the ENCODE project, we determined the histone modification profile of H3K4me3, H3K27me3, H3K9me3 in normal CD34+ and CD15+ cells. The observed profile was similar to that obtained in control leukocytes using ChIP targeting the WT1 5' interval (Figure 3A, B) suggesting very little cell-type variation in normal blood lineages. Unfortunately no ChIP-seq data is available for H3K4me2 for direct comparison. Subsequent experiments were performed on the $\mathrm{K} 562$ and NB4 cell lines that have methylated $A W T 1$ promoters, unmethylated WT1 CpG islands with high expression levels of the both transcripts. For these cells lines, the control GAPDH promoter and $\alpha$-satellite repeat regions were normal and comparable to control leukocytes with enrichment of the active H3K4me3 and repressive H3K9me3 marks, respectively. In both cell lines there was massive abundance of the active histone mark H3K4me3 at the WT1 promoter. This represents a change from modest H3K4me2 enrichment in control leukocytes that may directly reflect the high level of active transcription from this promoter. The $A W T 1$ promoter had epigenetically switched from an unmethylated interval with $\mathrm{H} 3 \mathrm{~K} 4 \mathrm{me} 2$ enrichment to having high levels of H3K9me3 in both K562 and NB4, a repressive mark functionally associated with DNA hypermethylation (Figure 3C, D) [11,12]. No H3K27me3 precipitation was observed at the $A W T 1$ or WT1 promoter regions in leukocyte or AML-derived cell lines (Figure 3B-D).

\section{The methylation profiles in cell lines reflect the epigenetic state in primary AML at diagnosis}

To ensure that the methylation profiles obtained in the hematological cancer cell lines could be recapitulated in primary hematological malignancies, we adapted our bisulphite PCRs for quantitative pyrosequencing and assessed a large cohort of cytogenetically well-characterized samples collected at initial diagnosis. These assays gave extremely consistent methylation values in our control cohort with low variability (WT1 average methylation 2.4\%, \pm 3SD (0-7.1\%); AWT1 average methylation $15.1 \%$, \pm 3SD (5.74-24.4\%). However we utilized a strict definition for hypermethylation of $>40 \%$ to ensure we identify only extreme cases of aberrant methylation. Using this criteria the unmethylated WT1 CpG island and hypermethylated $A W T 1$ signature was extremely prevalent in AML patients, including individuals with the recurrent genetic abnormalities inv(16)(p13.1q22), $\mathrm{t}(16 ; 16)(\mathrm{p} 13.1 ; \mathrm{q} 22)$ CBFB-MYH11, t(8;21)(q21;p22) AML1-ETO, t(15;17) (q22;q12) PML-RARA, t(9;11)(p22;q23) MLLT3-MLL, $\mathrm{t}(11 ; 10), \operatorname{inv}(3), \operatorname{inv}(9), N P M 1$ mutated and unspecified AML (Figure 4A, B). Of the 121 AML patients studied, only 12 samples did not reach this cut off, of which 6 (one inv(3), and 5 complex AML cases) presented with clear hypermethylation compared to controls but $>40 \%$ methylation which may reflect a lower blast count (Additional file 2: Table S1 and Additional file 3: Table S2). In contrast, only 2 samples had hypermethylation at the 


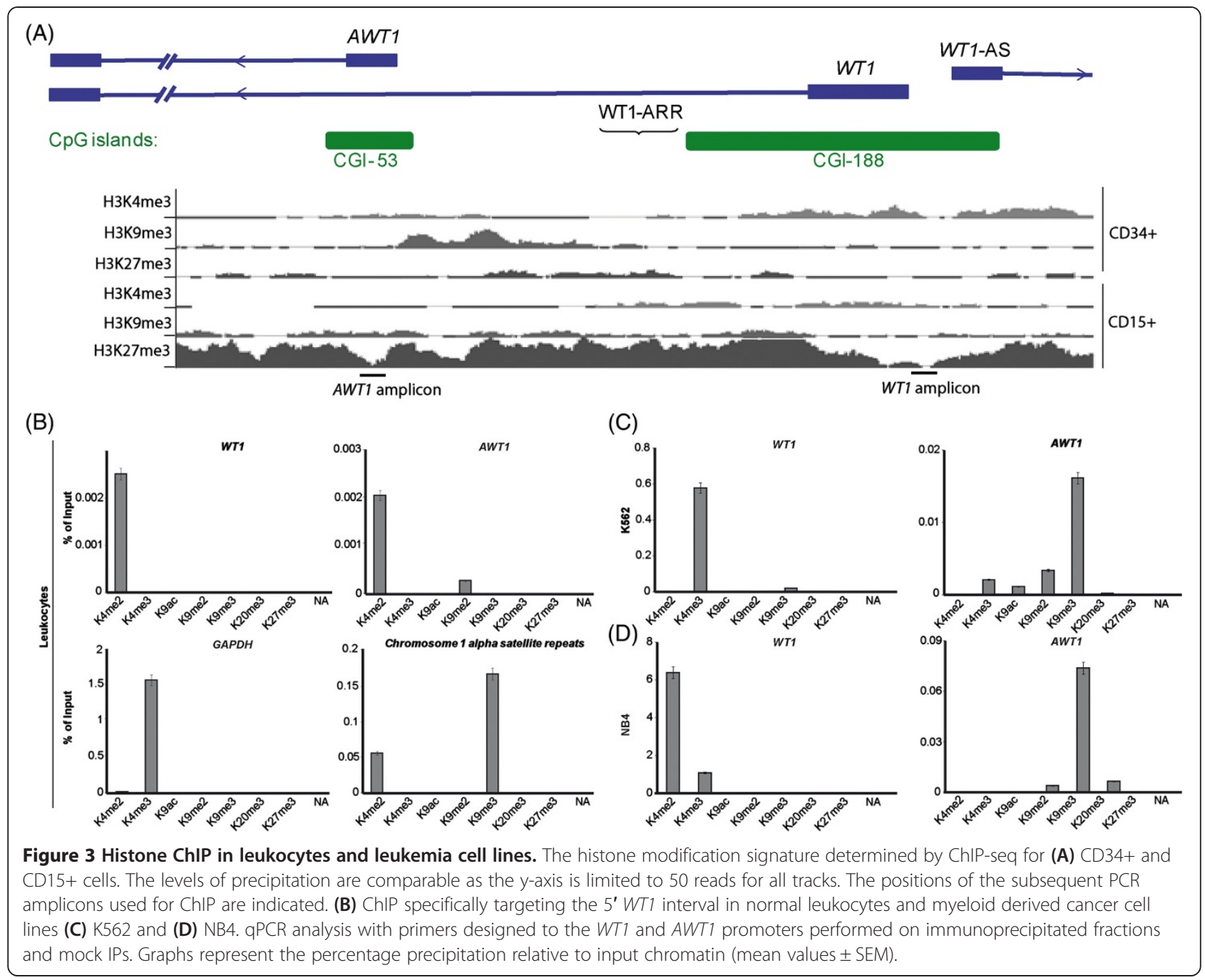

WT1 promoter, both complex cytogenetic AML cases. This analysis revealed that the hypermethylation status of the AWT1 promoter is an excellent marker, with a positive predictive value (PPV) of 100\%, indicating that this marker can correctly detect and classify healthy individual from those with AML. The sensitivity and negative predictive value (NPV) are $86.1 \%$ and $89.4 \%$ respectively (Additional file 2: Table S3).

\section{Methylation analysis in other primary hematological malignancies}

We extended our methylation analysis to other forms of hematological cancers. Similar to AML, chronic myeloid leukemia cases with BCR-ABL translocations had unmethylated WT1 promoters and AWT1 hypermethylation, however, polycythaemia vera and essential thrombocythaemia samples had methylation profiles indistinguishable from normal leukocyte controls. In samples classified with B-cell $t(9: 22)$ BCR/ABL, $t(4: 11)$ MLL/AFF1, $t(12: 21)$ TEL/ AML1 and T-cell lymphoblastic leukemias or follicular and Burkitt lymphomas we identified very variable methylation at the promoters, which was often significantly different from normal controls but not reaching the defined $40 \%$ methylation cut-off (Figure 4A, C).

\section{AWT1 hypermethylation discriminates AML patients who relapse from those achieving complete remission after SCT}

Relapse and progression remain the main causes of treatment failure in patients with AML after undergoing allogeneic SCT and often presents as refractory disease. Numerous reports have shown that $\sim 90 \%$ of patients with $A M L$ show WT1 overexpression, therefore, this transcript has been proposed as a useful marker for evaluating MRD after chemotherapy or SCT $[13,14]$. To determine whether methylation at the WT1 locus is a useful marker for disease monitoring we performed pyroseqeuncing for the WT1 and AWT1 promoters in a series of 9 AML patients before and after allogeneic SCT for whom WT1 expression had been previously determined [14]. All patients 


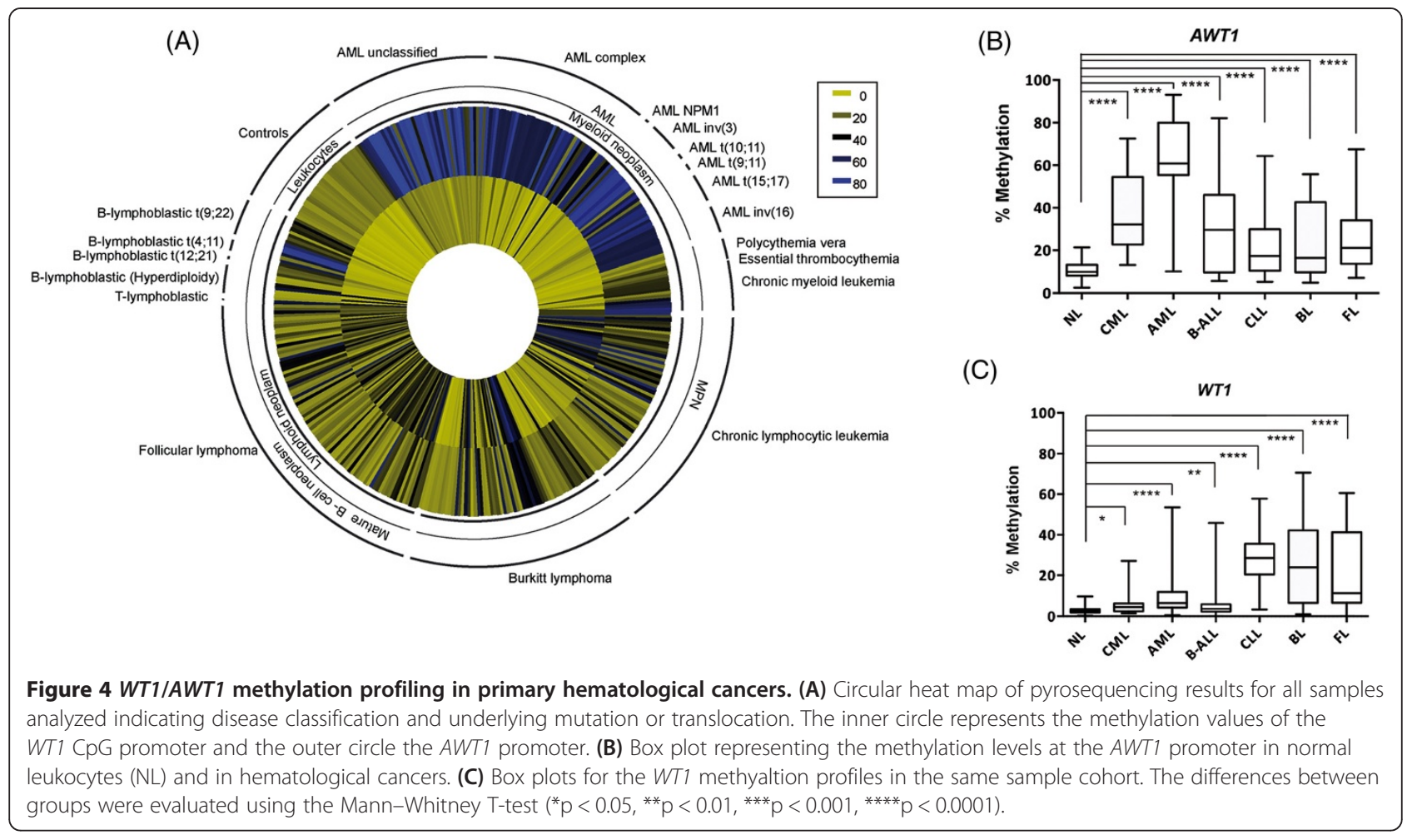

had positive WT1 expression as determine by RT-PCR at initial diagnosis or in pre-SCT bone marrow samples, of these 5 presented with disease relapse, of which 4 were associated with high WT1 levels. In 8 cases the $A W T 1$ promoter region was hypermethylated at initial assessment. During the post-transplantation period, high concordance between normal WT1 expression levels, AWT1 hypermethylation and remission status was seen in patients. Specifically, those patients who attained complete remission had WT1 expression below the cut-off, similar to normal controls, and the $A W T 1$ region remained unmethylated. In 4 of the 5 patients who underwent disease relapse showed overexpression of WT1 and gained methylation at the $A W T 1$ promoter, sometimes to levels higher than at initial diagnosis (Figure 5). Interestingly, the patient with low WT1 expression at relapse maintained the $A W T 1$ promoter in an unmethylated state post-SCT, despite being hypermethylated at diagnosis. Interestingly the WT1 CpG island remained unmethylated $(<13 \%$ methylation) in all patients and time points (data not shown).

\section{Discussion}

Recent studies have illustrated the power of methylation profiling to identify important cancer-related genes associated with the pathogenesis and classification of hematological cancers. For example $D B C 1$ hypermethylation is a prognostic marker for AML with normal karyotype [15]. In addition large-scale epigenetic studies have demonstrated that aberrant methylation is a hallmark of specific leukemia sub-types, often associated with known translocations or genetic mutations. This is best highlighted by the AML hypermethylation signature associated with $I D H 1 / I D H 2$ mutations, which disrupt TET2 function [16]. In our current study we observe extreme hypermethylation of the $A W T 1$ promoter, an epigenetic mark generally associated with robust gene silencing, in myeloid-derived hematological cancer cell lines and primary samples. These results are somewhat surprising as high expression levels of the various WT1 transcripts are frequently observed in AML $[17,18]$, a scenario that is normally mutually exclusive with abundant promoter methylation.

Loss-of-function mutations in TET2, an enzyme that converts $5 \mathrm{mC}$ to $5 \mathrm{hmC}$ have been observed in 8\% of AML cases [19]. Interrogation of the mutation status of TET2 in the Human Cancer Genome database revealed that Jurkat was the only cell in our panel that had pathological changes (p.Q831H). This suggests that absence of demethylation by mutated TET2 is not associated with the observed AWT1 hypermethylation.

Previous studies in gliomas and breast tumors have also revealed, contrary to expectation, a high frequency of WT1 expressing samples with promoter (CpG island $188)$ hypermethylation $[20,21]$. Interestingly one study assessed the affect of 5-aza-deoxycytidine on WT1 expression and observed that despite decreases in methylation there was not a concomitant change in WT1 expression [21]. Interestingly exposing the KG1A cell line to 

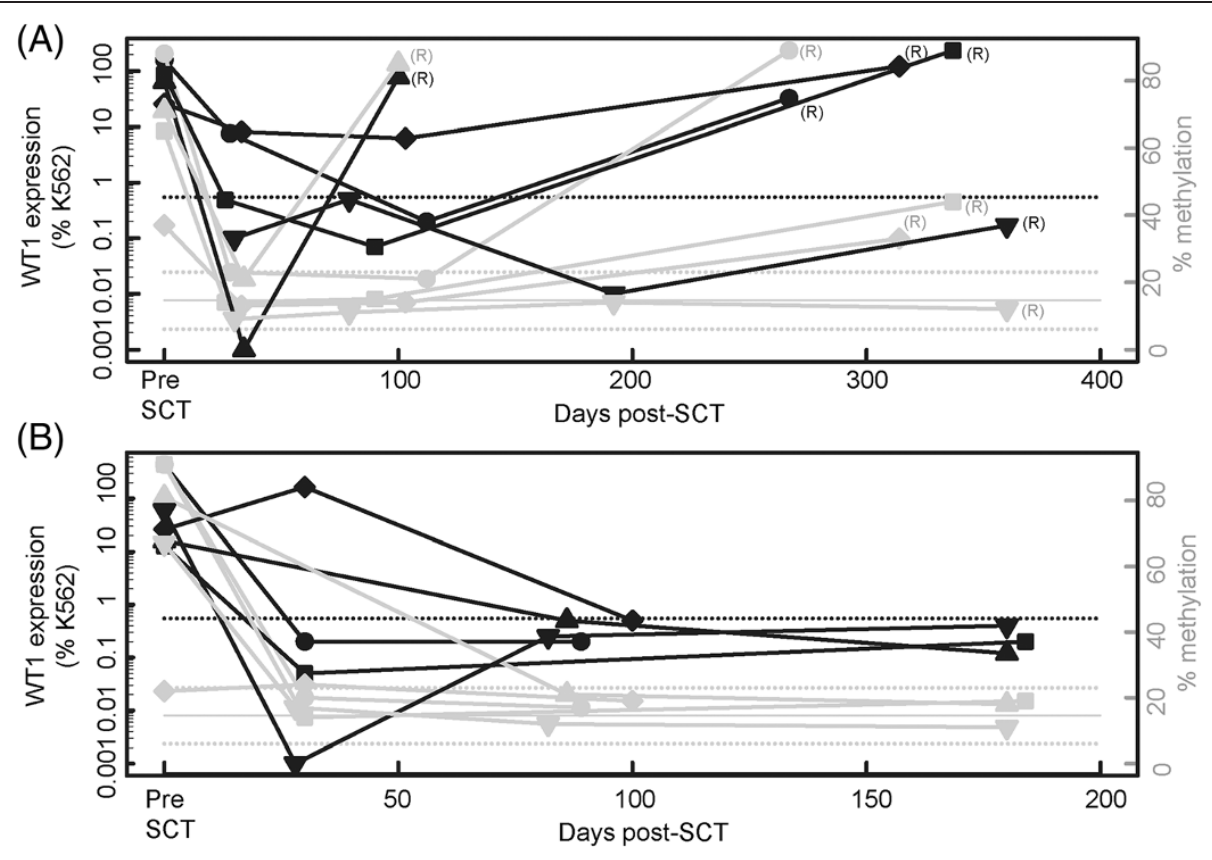

Figure 5 Time-course of AWT1 methylation after SCT. Evolution of AWT1 methylation and total WT1 expression in (A) patients who relapsed post-SCT $(n=5)$ and $(\mathbf{B})$ patients in complete remission $(n=4)$. Black lines represent expression levels relative to the K562 cell line, with the dashed black lines representing the pathological thresholds for expression (0.55\% of K562). The grey lines represent the methylation range observed in controls (14.6\% \pm 3 SD).

Decitabine resulted in drastic $(>50 \%)$ demethylation of the $A W T 1$ promoter but only a modest doubling in expression levels (Additional file 4: Figure S2). Despite being a specific cancer marker, the tumor-specific methylation at this promoter interval appears to be inconsequential to gene transcription and that other mechanism regulate expression. This implies that when interpreting DNA methylation patterns both cellular context and the genomic position of the CpG sites must be taken into consideration.

Our results in cell lines imply that the WT1 and $A W T 1$ isoforms are co-regulated, suggesting they share cis-acting regulatory elements. Recently an enhancer region located $\sim 1 \mathrm{~kb}$ past the shared 3'UTR has been identified and shown to bind the myeloid-specific transcription factor GATA-2 [22], with abundant expression of GATA-2 and WT1 in AML being associated with bad prognosis [23]. An expression analysis in the 26 of the hematological cancer cell lines and 53 primary AML samples revealed GATA-2 and WT1/AWT1 co-expressed in $96 \%$ cases $(51 / 56)$ consistent with this mechanism being a regulator of expression at the locus (Additional file 5: Figure S3). Computational analysis of the ENCODE dataset reveals that the GATA-2 site is enriched for H3K27ac, a histone modification frequently associated with enhancer elements [24]. This chromatin state may therefore be permissive to chromatin looping between this enhancer and the WT1 promoter region. Interrogation of the Chromatin Interaction Analysis Paired-End Tags (ChIA-PET) dataset in K562 cells suggests there is a strong physical association between these two genomic elements facilitated by POLII (Additional file 5: Figure S3).

Mutations in WT1 have been reported in $6-10 \%$ of AML cases, $20 \%$ of biphenotypic leukemias and sporadic T-cell acute lymphoblastic leukemias (T-ALL) [25-27]. This is a similar frequency to that found in sporadic Wilm's tumors [28,29]. In AML and T-ALL cases with heterozygous mutations, expression analyses have demonstrated biallelic expression of both the mutated and wild-type WT1 alleles consistent with our observations that the WT1 and AWT1 transcripts are not subject to genomic imprinting $[27,30]$. Interestingly, direct sequencing of all exons of the WT1 gene in our panel of cell lines identified only one mutation in addition to known polymorphisms, an $\mathrm{A}>\mathrm{G}$ nonsense mutation in exon 7 (p.R369*) of the non-expressing U937 cell line.

Despite the plethora of research on the role of WT1 in leukemia, it remains unclear if the abundant WT1 levels represent ectopic expression during carcinogenesis or simply reflects the arrested differentiation during early hematopoiesis, since most AML have an immature phenotype. However, the hypermethylation observed at this domain is associated with the tumorgenic changes since methylation at the WT1 and $A W T 1$ CpG islands is never 
observed in any normal hematopoietic cell type or CD34+ stem cells.

The discovery of novel molecular markers that are highly specific and sensitive will improve strategies for both detecting and classifying tumor types, but also in the management of cancer by monitoring a tumors response to novel therapies. Here we show that using a cut-off of $>40 \%$ methylation at the $A W T 1$ promoter, the PPV for our assay in AML is $100 \%$, indicating that the analysis can correctly detect and classifies a healthy individual. The sensitivity and NPV are $86.1 \%$ and $89.4 \%$ respectively, making this an ideal marker to correctly classify AML compared to controls. It must be noted, however, that when assessing all hematological malignancies together, hypermethylation of the AWT1 promoter it does not perform well as a diagnostic factor for unspecified leukemias/lymphomas (sensitivity $47 \%$; specificity $100 \%$; PPV 100\%; NPV 43.6\%) (Additional file 2: Table S3). Therefore quantitating $A W T 1$ hypermethyaltion is ideally suited to monitor MRD in which the diagnosis of AML has already been performed, and the recurrence of the disease during remission needs to be accurately monitored as revealed by our analysis of patients undergoing allogeneic SCT.

\section{Conclusion}

In summary, differences in methylation patterns among tumors may be correlated with clinical features of patients and can serve as markers in cancer classification. Here we describe a methylation signature of the $A W T 1$ promoter $\mathrm{CpG}$ island that is a promising marker for classifying myeloid-derived leukemias. When compared to the results of other well-characterized methylation markers in additional forms of cancer (i.e. MGMT in glioblastomas and GSTP1 in prostate cancers) [31,32] the outcomes are extremely encouraging and further work on larger cohorts, maybe in combination with other leukemia-specific methylation markers, are required to confirm if our observations can be useful in a clinical setting. It remains to be determined what mechanisms are responsible for the correlated up-regulation of WT1 and AWT1 expression, with DNA methylation, microRNA, enhancer activation or even utilization of an unidentified enhancing long non-coding RNA all possibly having an influence [33].

\section{Methods}

\section{Patient samples and cell lines}

DNA was extracted from frozen peripheral blood and/ or bone marrow samples from 356 patients at diagnosis and 102 control peripheral blood samples. For 9 individuals, we also had samples from multiple time points following SCT. Full clinical classification of the cohort can be found in Additional file 2: Table S1 and Additional file 3: Table S2. In addition, DNA and RNA were isolated from 28 derived from various hematological malignancies along with CD15+ (neutrophils and monocytes), CD19+ (B -cells) and CD3+ (T-lymphocyte) cell fractions. The cell lines were purchased directly from the ATCC or DSMZ cell line repositories and were grown in DMEM media with $10 \%$ heat inactivated fetal calf serum supplemented with $50 \mathrm{U} / \mathrm{ml}$ penicillin and $50 \mu \mathrm{g} / \mathrm{ml}$ streptomycin. In addition all cell lines were routinely tested to ensure they were free of mycoplasm contamination. These studies were approved by the ethical committee of Bellvitge Institute for Biomedical Research/CEIC (PR159/08) and all patients gave informed consent.

\section{Dataset analysis}

To determine the epigenetic landscape of the WT1 region we analyzed publically available H3K4me3, H3K9me3 and H3K27me3 ChIP-seq datasets and whole genome bisulphite sequencing (WGBS) methylomes for CD34+ and three leukocytes. All data analyses were performed using in-house R-scripts. We subsequently targeted specific regions of interest using bisulphite PCR.

\section{Methylation analysis}

Approximately $50 \mathrm{ng}$ of bisulphite converted DNA was used for each bisulphite PCR or pyrosequencing reaction (for primer sequences see Additional file 2: Table S4). To differentiate between 5 '-hydroxymethylation $(5 \mathrm{hmC})$ and 5-methylcytosine $(5 \mathrm{mC})$ we utilized methylated DNA immunoprecipitations (meDIP) with a specific $5 \mathrm{hmC}$ or $5 \mathrm{mC}$ antibodies (Diagenode, Liege, Belgium) and the Quest $5 \mathrm{hmC}$ Glucosyltransferase Detection KitTM (Zymo).

\section{Chromatin immunoprecipitations}

For ChIP, $100 \mu \mathrm{g}$ of chromatin was used for each immunoprecipitation reaction with Protein A Agarose/Salmon Sperm DNA and specific antibody raised against various post-translational histone $\mathrm{H} 3$ or $\mathrm{H} 4$ modifications (H3K4me2 Millipore 07-030; H3K4me3 Millipore 07473; H3K9ac Cell Signalling 9671; H3K9me2 Millipore 07-441; H3K9me3 Abcam 8898; H4K20me3 Millipore 07-463; H3K27me3 Millipore 07-449). The levels of immunoprecipitated chromatin at specific regions were determined by qPCR using an Applied Biosystems 7900 Fast real-time PCR machine, using SYBR Green and IP levels were compared to Input and the results expressed as percentage of total input material.

\section{Quantitative RT-PCR}

The levels of various WT1 transcripts were assessed using qRT-PCR. All PCR amplifications were run in triplicate on a 7900 Fast real-time PCR machine (Applied Biosystems) following the manufacturers' protocol. All primers were optimized using SYBR Green (Additional 
file 2: Table S4 for primer sequences) and melt curve analysis to ensure that amplicons were specific and free of primer-dimer products. Thermal cycle parameters included Taq polymerase activation at $95^{\circ} \mathrm{C}$ for $10 \mathrm{~min}$ for 1 cycle, repetitive denaturation at $95^{\circ} \mathrm{C}$ for $15 \mathrm{sec}$, and annealing at $60^{\circ} \mathrm{C}$ for $1 \mathrm{~min}$ for 40 cycles. All resulting triplicate cycle threshold $(\mathrm{Ct})$ values had to be with $1 \mathrm{Ct}$ of each other. The quantitative values for each triplicate were determined as a ratio with the level of $\beta$-Actin expression that was measured in the same sample, and then averaged to provide relative expression values. A cDNA mix of all cell lines and controls was used as an expression calibrator.

\section{Western blot analysis}

We extracted nuclear and cytoplasm protein fractions from cell lines for western blot using the NE-PER Nuclear and Cytoplasmic Extraction kit (Thermo Scientific). SDSPAGE gel electrophoresis and western blotting was performed as standard. To determine the cellular localization of proteins we incubated resulting membranes with primary antibodies against WT1 (Santa Cruz sc-192), $\beta$-Tubulin (Abcam ab6046) or Nucleolin (Santa Cruz sc-8031). Washed membranes were incubated with corresponding peroxidase-conjugated secondary antibody. The immunoreactive proteins were visualized using the ECL Western blotting detection kit (Amersham Biosciences).

\section{Additional files}

Additional file 1: Figure S1. (A) Confirmation of imprinted methylation at the H19-DMR in peripheral leukocytes. (B) Bisulphite PCR analysis of the WT1 promoter interval in cell lines derived from hematological cancers other than AML.

Additional file 2: Table S1. Characterization of hematological neoplasms according to cytogenetic aberrations. Table S3. Specificity of AWT1MT1 hypermethylation as a biomarker. Table S4. The PCR primer sequences used in this study.

Additional file 3: Table S2. Characterization of hematological neoplasms using WHO classification with information on age and sex of the patients (if data available). The methylation values obtained following pyrosequencing for the WT1 and AWT1 promoters are also given.

Additional file 4: Figure S2. (A) Methylation values of repetitive DNA elements in the KG1A cell lines treated with different concentrations of Decitabine. (B) The methylation values for WT1 and AWT1 promoters and (C) qRT-PCR for WT1, AWT1 and WT1-AS transcripts following Decitabine treatment.

Additional file 5: Figure S3. (A) Map of the WT1 locus showing POL mediated ChIA-PET interactions between the GATA-2 enhancer and the WT1 promoter interval. (B) Abundance of the GATA-2 transcription factor in hematological cancer cell lines as determined by qRT-PCR.

\section{Abbreviations}

WT1: Wilms tumour 1 gene; AML: Acute myeloid leukemia; MRD: Minimal residual disease; SCT: Stem cell transplant; RT-PCR: Reverse transcriptase polymerase chain reaction; ChIP: Chromatin immunoprecipitation; $5 \mathrm{hmC}$ : 5- hydroxymethylation; 5mC: 5-methylcytosine.
Competing interests

The authors declare that they have no competing interests.

\section{Authors' contributions}

AMA carried out the molecular studies and FC performed the bioinformatic analyses. JR, MDO, JS, ZA, AC, ME FP, MJC, IB, MK, RS provided reagents and classified patients. AMA, RS and DM conceived of the study, and participated in its design and coordination and helped to draft the manuscript. All authors read and approved the final manuscript.

\section{Acknowledgements}

Authors would like to thank Marta Kulis and Carles Arribas for help with pyrosequencing and we would like to thank all the patients and their families for participating in this project. This work was partially funded by European Community's Seventh Framework Programme (FP7/2007-2013) under the HEALTH-F5-2011-282510 -BLUEPRINT (to M.E) and Spanish Ministerio de Educacion y Ciencia (BFU2011-27658 to D.M). D.M is a Ramon y Cajal research fellow and A.G.A was funded by a FPU studentship and was awarded an EMBO short-term fellowship to undertake part of this work.

\section{Author details}

${ }^{1}$ Imprinting and Cancer group, Cancer Epigenetic and Biology Program, Institut d'Investigació Biomedica de Bellvitge, Hospital Duran i Reynals, Av. Gran Via de L'Hospotalet 199-203, 08907 L'Hospitalet de Llobregat, Barcelona, Spain. ${ }^{2}$ Institute of Human Genetics, Christian-Albrechts University, Kiel, Germany. ${ }^{3}$ Department of Genetics, University of Navarra, Pamplona, Spain. ${ }^{4}$ Cancer Epigenetics group, Cancer Epigenetic and Biology Program, Institut d'Investigació Biomedica de Bellvitge, Hospital Duran i Reynals, Barcelona, Spain. ${ }^{5}$ Division of Cancer and Area of Cell Therapy and Hematology Service, Universidad de Navarra, Pamplona, Spain. ${ }^{6}$ Servicio de Heamatología, Hospital Sant Joan de Déu, Barcelona, Spain. ${ }^{7}$ Department of Physiological Sciences II, School of Medicine, University of Barcelona, Barcelona, Catalonia, Spain. Institucio Catalana de Recerca i Estudis Avançats (ICREA), Barcelona, Catalonia, Spain. ${ }^{9}$ Department of Hematology, Hospital General Universitario Gregorio Maranon, Madrid, Spain.

Received: 15 November 2013 Accepted: 19 December 2013 Published: 9 January 2014

\section{References}

1. Baird PN, Simmons PJ: Expression of the Wilms' tumor gene (WT1) in normal hemopoiesis. Exp Hematol 1997, 25:312-320.

2. Hohenstein P, Hastie ND: The many facets of the Wilms' tumour gene, WT1. Hum Mol Genet 2006, 15 Spec:R196-R201.

3. Dallosso AR, Hancock AL, Brown KW, Williams AC, Jackson S, Malik K: Genomic imprinting at the WT1 gene involves a novel coding transcript (AWT1) that shows deregulation in Wilms' tumour. Hum Mol Genet 2004, 13:405-415.

4. Mitsuya K, Sui H, Meguro M, Kugoh H, Jinno Y, Niikawa N, Oshimura M: Paternal expression of WT1 in human fibroblasts and lymphocytes. Hum Mol Genet 1997, 6:2243-2246.

5. Dallosso AR, Hancock AL, Malik S, Salpekar A, King-Underwood L, Pritchard-Jones K, Peters J, Moorwood K, Ward A, Malik KT, Brown KW: Alternately spliced WT1 antisense transcripts interact with WT1 sense RNA and show epigenetic and splicing defects in cancer. RNA 2007, 13:2287-2299.

6. Malik K, Salpekar A, Hancock A, Moorwood K, Jackson S, Charles A, Brown KW: Identification of differential methylation of the WT1 antisense regulatory region and relaxation of imprinting in Wilms' tumor. Cancer Res 2000, 60:2356-2360.

7. Nomdedéu JF, Hoyos M, Carricondo M, Bussaglia E, Estivill C, Esteve J, Tormo M, Duarte R, Salamero O, de Llano MP, García A, Bargay J, Heras I, Martí-Tutusaus JM, Llorente A, Ribera JM, Gallardo D, Aventin A, Brunet S, Sierra J: Bone marrow WT1 levels at diagnosis, post-induction and postintensification in adult de novo AML. Leukemia 2013, 27:2157-2164.

8. Andersson C, Li X, Lorenz F, Golovleva I, Wahlin A, Li A: Reduction in WT1 gene expression during early treatment predicts the outcome in patients with acute myeloid leukemia. Diagn Mol Pathol 2012, 21:225-233.

9. Matsuo Y, Ariyasu T, Ohmoto E, Kimura I, Minowada J: Bi-phenotypic t (9;22)-positive leukemia cell lines from a patient with acute leukemia: NALM-20, established at the onset; and NALM-21, NALM-22 and NALM-23, established after relapse. Hum Cell 1997, 4:335-358. 
10. Wu H, D'Alessio AC, Ito S, Wang Z, Cui K, Zhao K, Sun YE, Zhang Y: Genome-wide analysis of 5-hydroxymethylcytosine distribution reveals its dual function in transcriptional regulation in mouse embryonic stem cells. Genes Dev 2011, 25:679-684.

11. Ohm JE, McGarvey KM, Yu X, Cheng L, Schuebel KE, Cope L, Mohammad HP, Chen W, Daniel VC, Yu W, Berman DM, Jenuwein T, Pruitt K, Sharkis SJ, Watkins DN, Herman JG, Baylin SB: A stem cell-like chromatin pattern may predispose tumor suppressor genes to DNA hypermethylation and heritable silencing. Nat Genet 2007, 39:237-242.

12. McGarvey KM, Van Neste L, Cope L, Ohm JE, Herman JG, Van Criekinge W, Schuebel KE, Baylin SB: Defining a chromatin pattern that characterizes DNA-hypermethylated genes in colon cancer cells. Cancer Res 2008, 68:5753-5759.

13. Bergmann L, Miething C, Maurer U, Brieger J, Karakas T, Weidmann E, Hoelzer D: High levels of Wilms' tumor gene (wt1) mRNA in acute myeloid leukemias are associated with a worse long-term outcome. Blood 1997, 90:1217-1225.

14. Kwon M, Martínez-Laperche C, Infante M, Carretero F, Balsalobre P, Serrano D, Gayoso J, Perez-Corral A, Anguita J, Diez-Martin JL, Buno L: Evaluation of minimal residual disease by real-time quantitative PCR of Wilms' tumor 1 expression in patients with acute myelogenous leukemia after allogeneic stem cell transplantation: correlation with flow cytometry and chimerism. Biol Blood Marrow Transplant 2012, 18:1235-1242

15. Alvarez S, Suela J, Valencia A, Fernández A, Wunderlich M, Agirre X, Prosper F, Martin-Subero Jl, Maiques A, Acquardo F, Rodriguez Perales S, Calasanz MJ, Roman-Gómez J, Siebert R, Mulloy JC, Cervera J, Sanz MA, Esteller M, Cigudosa JC: DNA methylation profiles and their relationship with cytogenetic status in adult acute myeloid leukemia. PLoS One 2010, 5:e12197.

16. Figueroa ME, Abdel-Wahab O, Lu C, Ward PS, Patel J, Shih A, Li Y, Bhagwat N, Vasanthekumar A, Fernandez HF, Tallman MS, Sun Z, Wolniak K, Peeters JK, Liu W, Choe SE, Fantin VR, Paietta E, Löwenberg B, Licht JD, Godley LA, Delwel R, Valk PJ, Thompson CB, Levine RL, Melnick A: Leukemic IDH1 and $\mathrm{IDH} 2$ mutations result in a hypermethylation phenotype, disrupt TET2 function, and impair hematopoietic differentiation. Cancer Cell 2010, 18:553-567.

17. Siehl JM, Reinwald M, Heufelder K, Menssen HD, Keliholz U, Thiel E: Expression of Wilm's tumor gene 1 at different stages of acute myeloid leukemia and analysis of its major splice variants. Ann Hematol 2004, 83:745-750.

18. Inoue K, Ogawa H, Sonoda Y, Kimura T, Sakabe H, Oka Y, Miyake S, Tamaki H, Oji Y, Yamagami T, Tatekawa T, Soma T, Kishimoto T, Sugiyama H: Aberrant overexpression of the Wilms tumor gene (WT1) in human leukemia. Blood 1997, 89:1405-1412.

19. Delhommeau F, Dupont S, Della Valle V, James C, Trannoy S, Massé A, Kosmider O, Le Couedic JP, Robert F, Alberdi A, Lécluse Y, Plo I, Dreyfus FJ, Marzac C, Casadevall N, Lacombe C, Romana SP, Dessen P, Soulier J, Viguié F, Fontenay M, Vainchenker W, Bernard OA: Mutation in TET2 in myeloid cancers. N Engl J Med 2009, 360:2289-2301.

20. Loeb DM, Evron E, Patel CB, Sharma PM, Niranjan B, Buluwela L, Weitzman SA, Korz D, Sukumar S: Wilms' tumor suppressor gene (WT1) is expressed in primary breast tumors despite tumor-specific promoter methylation. Cancer Res 2001, 61:921-925.

21. Costello JF, Frühwald MC, Smiraglia DJ, Rush L, Robertson GP, Gao X, Wright FA, Feramisco JD, Peltomaki P, Lang JC, Schuller DE, Yu L, Bloomfield CD, Caligiuri MA, Yates A, Nishikawa R, Su Huang H, Petrelli NJ, Zhang X, O'Dorisio MS, Held WA, Cavenee WK, Plass C: Aberrant CpG-island methylation has non-random and tumour-type-specific patterns. Nat Genet 2000, 24:132-138.

22. Furuhata A, Murakami M, Ito H, Gao S, Yoshida K, Sobue S, Kikuchi R, Iwasaki T, Takagi A, Kojima T, Suzuki M, Abe A, Naoe T, Murate T: GATA-1 and GATA-2 binding to 3' enhancer of WT1 gene is essential for its transcription in acute leukemia and solid tumor cell lines. Leukemia 2009, 23:1270-1277.

23. Vicente C, Vazquez I, Conchillo A, García-Sánchez MA, Marcotegui N, Fuster O, Gonzalez M, Calasanz MJ, Lahortiga I, Odero MD: Overexpression of GATA2 predicts an adverse prognosis for patients with acute myeloid leukemia and it is associated with distinct molecular abnormalities. Leukemia 2012, 26:550-554.
24. Rada-lglesias A, Bajpai R, Swigut T, Brugmann SA, Flynn RA, Wysocka JA: Unique chromatin signature uncovers early developmental enhancers in humans. Nature 2011, 470:279-283.

25. The Cancer Genome Atlas Research Network: Genomic and epigenomic landscape of adult de novo acute myeloid leukemia. N Engl J Med 2013, 368:98.

26. Carapeti M, Goldman JM, Cross NC: Dominant-negative mutations of the Wilms' tumour predisposing gene (WT1) are infrequent in CML blast crisis and de novo acute leukaemia. Eur J Haematol 1997, 58:346-349.

27. King-Underwood L, Pritchard-Jones K: Wilms' tumor (WT1) gene mutations occur mainly in acute myeloid leukemia and may confer drug resistance. Blood 1998, 91:2961-2968.

28. Schumacher V, Schärer K, Wühl E, Altrogge H, Bonzel KE, Guschmann M, Neuhaus TJ, Pollatro RM, Kuwertz-Broking E, Bulla M, Tondera AM, Mundel P, Helmchen U, Waldherr R, Weirich A, Royer-Pokora B: Spectrum of early onset nephrotic syndrome associated with WT1 missense mutations. Kidney Int 1998, 53:1594-1600.

29. Little SE, Hanks SP, King-Underwood L, Jones C, Rapley EA, Rahman N, Pritchard-Jones K: Frequency and heritability of WT1 mutations in nonsyndromic Wilms' tumor patients: a UK Children's Cancer Study Group Study. J Clin Oncol 2004, 22:4140-4146.

30. Tosello V, Mansour MR, Barnes K, Paganin M, Sulis ML, Jenkinson S, Allen CG, Gale RE, Linch DC, Palomero T, Real P, Murty V, Yao X, Richards SM, Goldstone A, Rowe J, Basso G, Wiernik PH, Paietta E, Pieters R, Horstmann M, Meijerink JP, Ferrando AA: WT1 mutations in T-ALL. Blood 2009, 114:1038-1045.

31. Hegi ME, Diserens AC, Gorlia T, Hamou MF, de Tribolet N, Weller M, Kros JM, Hainfellner JA, Mason W, Mariani L, Bromberg JE, Hau P, Mirimanoff RO, Cairncross JG, Janzer RC, Stupp R: MGMT gene silencing and benefit from temozolomide in glioblastoma. N Engl J Med 2005, 352:997-1003.

32. Hopkins TG, Burns PA, Routledge MN: DNA methylation of GSTP1 as biomarker in diagnosis of prostate cancer. Urology 2007, 69:11-16.

33. Ørom UA, Shiekhattar R: Long noncoding RNAs usher in a new era in the biology of enhancers. Cell 2013, 154:1190-1193.

doi:10.1186/1756-8722-7-4

Cite this article as: Guillaumet-Adkins et al:: Hypermethylation of the alternative AWT1 promoter in hematological malignancies is a highly specific marker for acute myeloid leukemias despite high expression levels. Journal of Hematology \& Oncology 2014 7:4.

\section{Submit your next manuscript to BioMed Central and take full advantage of:}

- Convenient online submission

- Thorough peer review

- No space constraints or color figure charges

- Immediate publication on acceptance

- Inclusion in PubMed, CAS, Scopus and Google Scholar

- Research which is freely available for redistribution 